\title{
Comparison of Gradients in Orientation and Stress between Experiment and Simulation
}

\author{
Anthony D. Rollett ${ }^{\mathrm{a}}$, Francis Wagner ${ }^{\mathrm{b}}$, Nathalie Allain-Bonasso ${ }^{\mathrm{c}}$, \\ David P. Field ${ }^{d}$, Ricardo A. Lebensohn ${ }^{\mathrm{e}}$ \\ aMaterials Sci. \& Eng., Carnegie Mellon Univ., Pittsburgh PA, USA \\ b,c LEM3, Univ. of Metz, Metz, France \\ 'School of Mech. \& Materials Eng., Washington State Univ., Pullman, WA, USA \\ 'Los Alamos National Laboratory, NM, USA \\ arollett@andrew.cmu.edu, brancis.wagner@univ-metz.fr, 'Nathalie.allain-bonasso@univ-metz.fr \\ dfield@mme.wsu.edu, ${ }^{e}$ lebenso@lanl.gov
}

Keywords: Orientation gradients, texture, simulation, FFT

\begin{abstract}
We examine the relationship between local gradients in orientation, which are quantified with the Kernel Average Misorientation, and the grain boundary network in an interstitial-free steel sheet, before and after $12 \%$ tensile strain. A portion of the unstrained microstructure is used as input to a full-field spectral viscoplastic code that simulates the same deformation. The orientation gradients are concentrated near grain boundaries in both experiments and simulation. Mapping out stress gradients in the simulation suggests that the development of orientation gradients is strongly correlated with such gradients.
\end{abstract}

\section{Introduction}

This paper seeks to quantify the concentration of (local) orientation gradients near to microstructural features such as grain boundaries in an IF-steel. Full field viscoplastic modeling of the tensile test is employed to verify the trends observed experimentally. There is a rich literature on orientation gradients in polycrystalline materials that goes all the way back to studies of the development of asterism during plastic deformation e.g. [1]. Ashby's suggestion of the necessity for geometrically necessary dislocations [2] has prompted intense interest in orientation gradients in deformed polycrystals especially with respect to quantification of the Nye tensor [3]. This paper represents an attempt to link the development of orientation gradients with the presence of grain boundaries and also with the existence of gradients in stress during deformation. Other papers in this same conference proceedings address more details of the analysis of the experimental effort.

\section{Materials, Methods}

The material tested was an interstitial-free (IF) steel with thickness $0.7 \mathrm{~mm}$. Calculations were performed using a standard viscoplastic model for crystal plasticity, Eq. 1, embedded in a code, VPFFT, that solves the equations of stress equilibrium and strain compatibility in a periodic unit cell built from a two- or three-dimensional image of the material. In the equation given, $\mathrm{D}$ is the (imposed) deviatoric strain rate, $\dot{\gamma}_{0}$ is a reference slip rate, $\boldsymbol{m}$ is a slip tensor (outer product of the slip direction and slip plane normal) for the $s^{\text {th }}$ slip system, $\tau$ is the corresponding critical resolved shear stress, $n$ is the corresponding exponent, and $\sigma$ is the deviatoric stress. For a strain rate imposed to the unit cell, the strain rate and stress fields and the corresponding effective stress are obtained iteratively. The code relies on the Fast Fourier transform (FFT) to alternate between real and frequency spaces. This permits convolution integrals to be replaced by point-wise tensor products (in frequency space), thus accelerating the computation. The method is based on the work of Moulinec \& Suquet on elastic composites [4] and the viscoplastic method has been described in several previous publications, e.g. Lebensohn [5] and Lebensohn et al. [6]. Work on misorientation development in copper, Lebensohn et al. [7] demonstrated the ability of the code to reproduce subgrain development. Analysis of hot spots in stress, Rollett et al. [8], showed that the hot spots tend to occur near grain boundaries. 


$$
D_{i j}=\dot{\gamma}_{0} \sum_{s} m_{i j}^{(s)}\left|\frac{m_{k l}^{(s)} \sigma_{k l}}{\tau^{(s)}}\right|^{n^{(s)}} \operatorname{sgn}\left(m_{k l}^{(s)} \sigma_{k l}\right)
$$

\section{Results}

An area with dimensions 1256 by 1011 pixels was mapped using electron back-scatter diffraction in a JEOL 6500F FEG-SEM scanning electron microscope. Fig. 1a shows a micrograph colored according to the crystal plane exposed at the surface (so-called normal direction inverse pole figure map, ND-IVP). The same area within a few microns was re-mapped (1255 by 911 ) after a $12 \%$ tensile strain had been applied, Fig. 1b. The color scheme is the standard one with red denoting $\{100\}$, blue $\{111\}$ and green $\{110\}$.

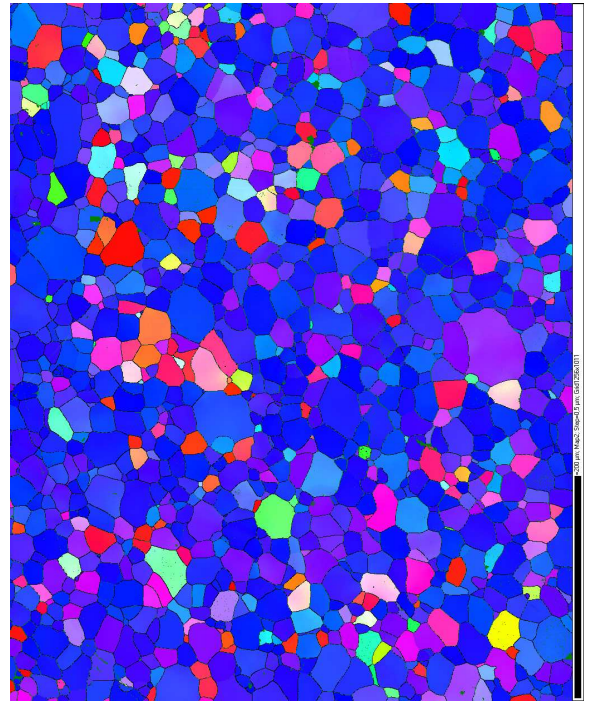

Fig. 1a. ND-IPF map of specimen of IF steel, as-received condition. The step size in both this EBSD map and that shown in Fig. $1 b$, is 0.5 microns.

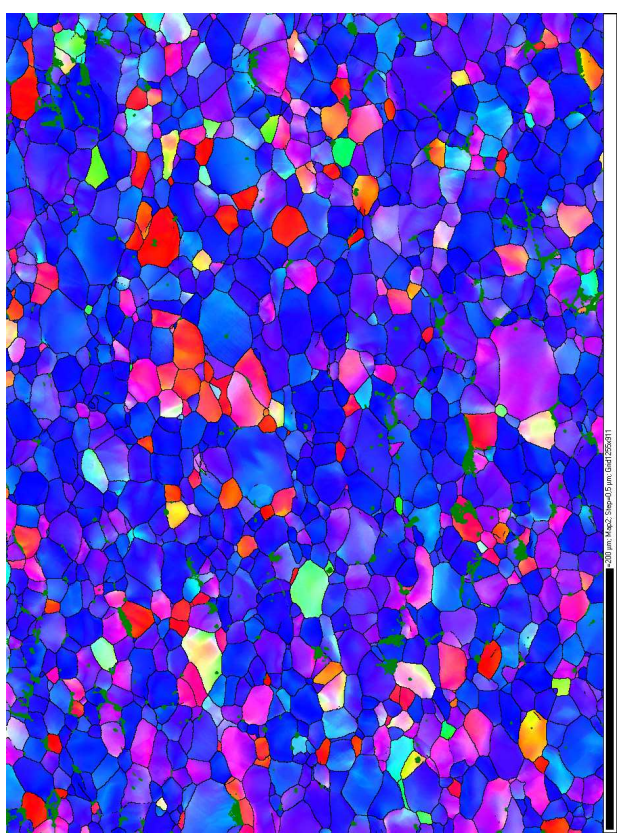

Fig. 1b. ND-IPF map of specimen of IF steel, after $12 \%$ tensile strain parallel to the vertical direction in the figure. Variations in color show that intra-grain orientation gradients have developed.

The same orientation maps were analyzed for kernel average misorientation (KAM), which is computed in the usual way with a threshold of $5^{\circ}$. The threshold ensures that high-angle boundaries do not give artificially high values for points adjacent to boundaries. The two maps are shown in Fig. 2a, un-strained, and Fig. 2b, after 12\% strain. They are colored on similar scales and it is apparent that significant intra-grain orientation gradients have developed in the strained sample; the scale is smaller in the undeformed map in order to make orientation gradients apparent. Note that previous work has focused on line scans of the gradients within individual grains, e.g. [9].

Approximately one quarter of the area was selected from the bottom left of the microstructure shown in Fig. 1a, with pixel dimensions 512 by 512 and used as input to the VPFFT code. The plane section input was extruded for four layers (in the absence of information into the depth of the material) so that the grid used was $512 \times 512 \times 4$. The code was run on a desktop with eight cores, which required about thirty minutes for twelve steps of $1 \%$ tensile strain. Fig. 3a shows an ND-IVP map for the simulated area; the color scheme is similar to that used in Fig. 1a. Fig. 3b shows the map of the von Mises equivalent stress, in which it is apparent that there are appreciable differences between the grains. In order to understand the development of orientation gradients, gradients in each component of the stress were computed. Since the VPFFT code transforms the stress field into Fourier (frequency) space at each iteration, it was straightforward to compute the first spatial 
derivative for each component since, given a differentiable function $f(x)$, with Fourier Transform $F(\xi)$, then the Fourier transform of the derivative of the function is $2 \pi i \xi F(\xi)$. In terms of computations on a grid, the value of the transform at each point is multiplied by the associated wave vector, transposed between the real and imaginary parts, and anti-transformed. Limiting our search to correlations between scalar variables, the tensor valued stress gradient was reduced to the root mean square value, in a manner analogous to working with the (scalar) von Mises equivalent stress. Figs. $3 \mathrm{c}$ and $3 \mathrm{~d}$ show the local average misorientation map and the magnitude of the gradient of the stress (tensor) field, respectively. The two fields, while not one to one, have a striking similarity.

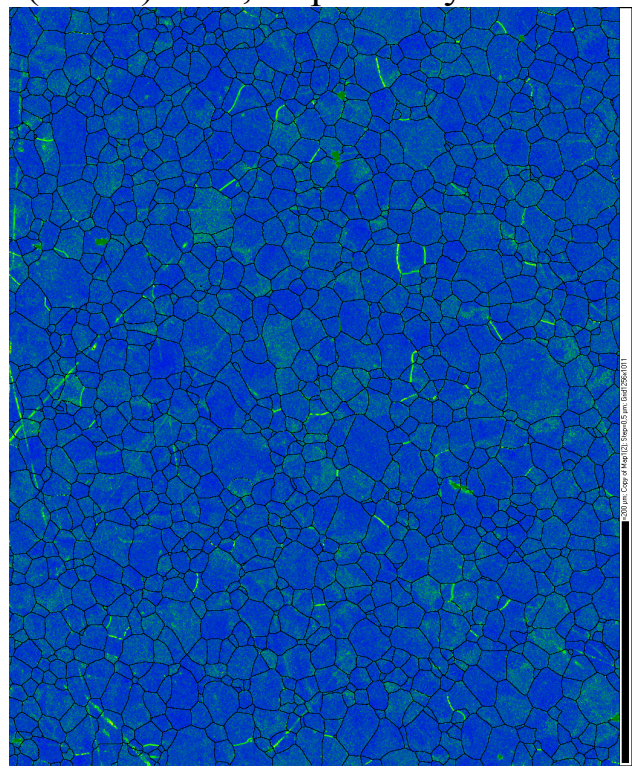

Fig. 2a. Map of local (kernel) average misorientation (KAM), as-received condition. The gradation in color from blue to red corresponds to misorientations (degrees) between 0.25 and $0.5^{\circ}$.

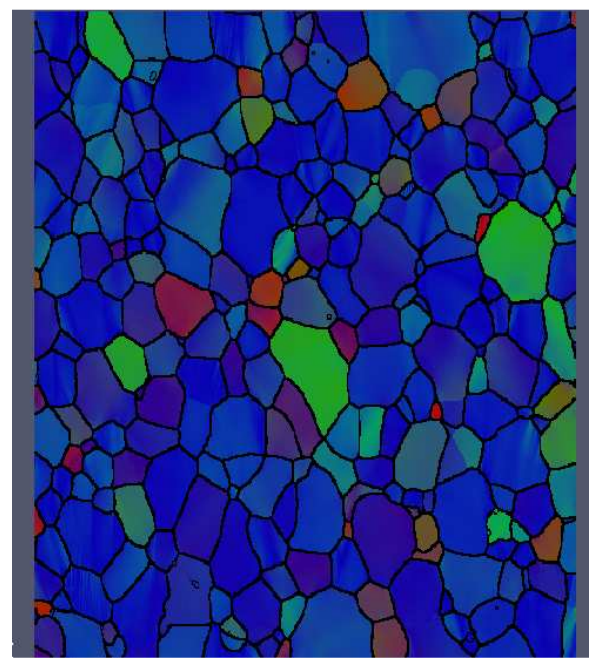

Fig. 3a. Excerpt from the as-received microstructure used to instantiate the simulation of the tensile test. The subset of the original image was taken from the lower left corner of Fig. 1a.

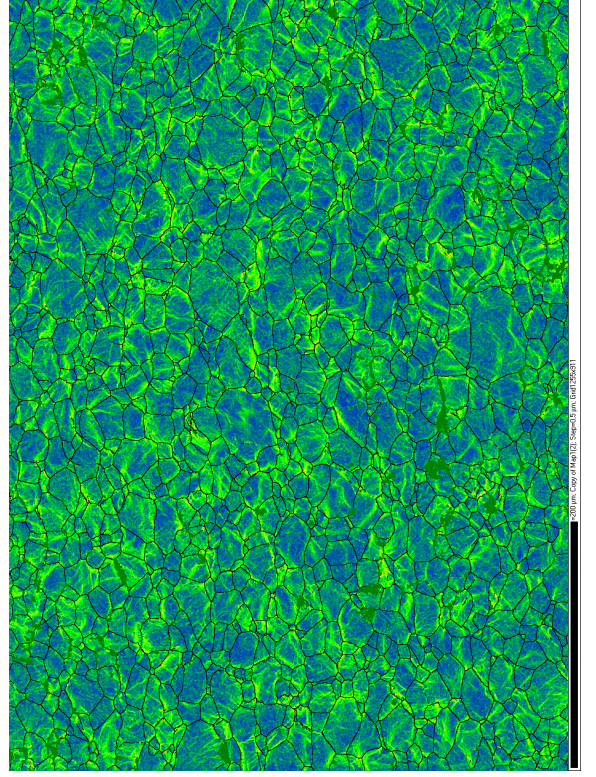

Fig. 2b. Map of KAM, after $12 \%$ tensile strain parallel to the vertical direction in the figure. The gradation in color from blue to red corresponds to misorientations (degrees) between 0.5 and $1.0^{\circ}$.

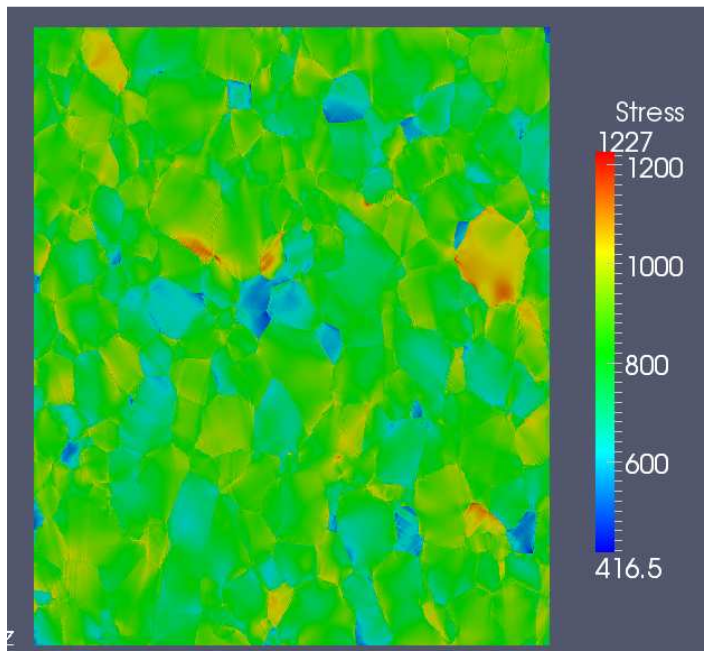

Fig. 3b. Stress (von Mises equivalent) field for the simulated tension test (12\% strain). 


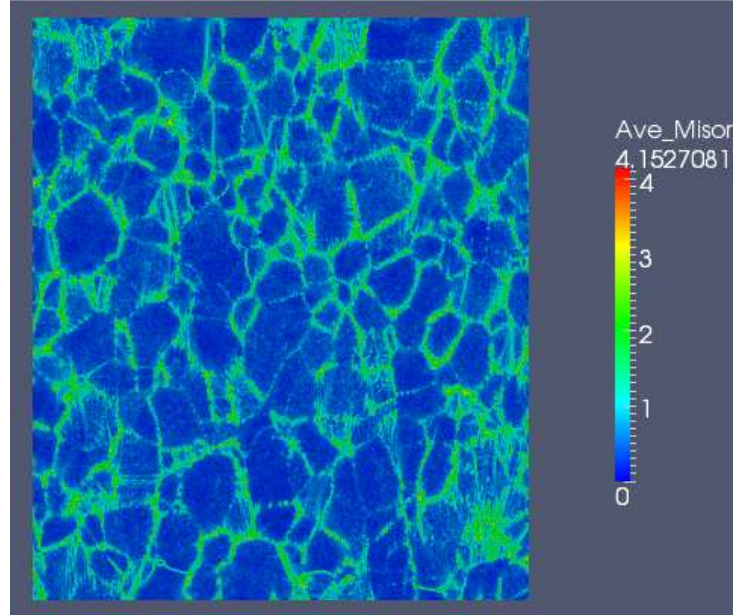

Fig. 3c. Local Average Misorientation (KAM) for the simulated tension test $(12 \%$ strain).

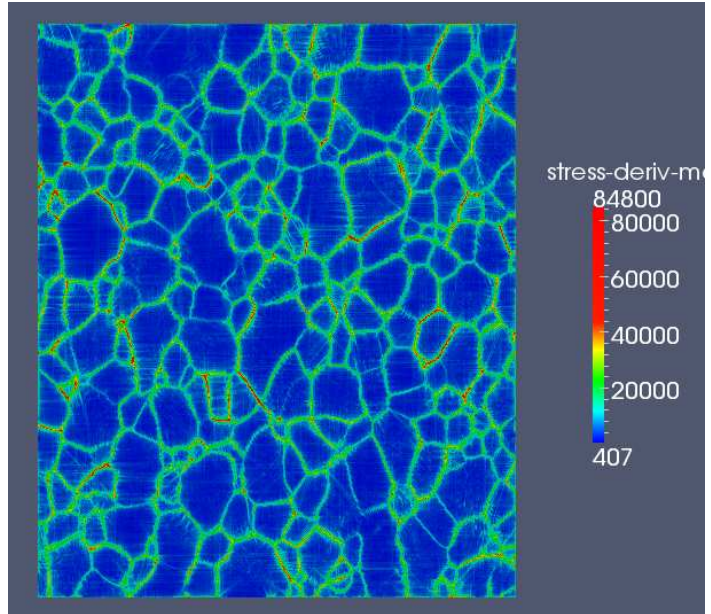

Fig. 3d. Magnitude of the stress gradient (at $12 \%$ strain). Contours have been adjusted to emphasize smaller values.

\section{Discussion}

The results shown offer direct evidence that nearness to a grain boundary promotes the development of orientation gradients. Pointwise correlation plots can obscure relationships, however, especially in non-linear cases where there is significant scatter. First, the Euclidean distance map [10] was computed for each microstructure, based on distance from the nearest grain boundary. This was found to be useful in a previous study of hot spots in stress [8]. A simple procedure is to choose two variables and bin the data (for each point) by one variable while making an average of the other variable for each bin. This was done for local average misorientation (KAM) and grain boundary distance. Fig. 4a shows that the KAM decreases essentially monotonically with distance from a grain boundary. Error bars denote one standard deviation. Conversely Fig. $4 \mathrm{~b}$ shows the same inverse relationship but now plotting averaged distance against KAM value. There is a small uptick at large values in both cases, the reason for which is not clear at this point.

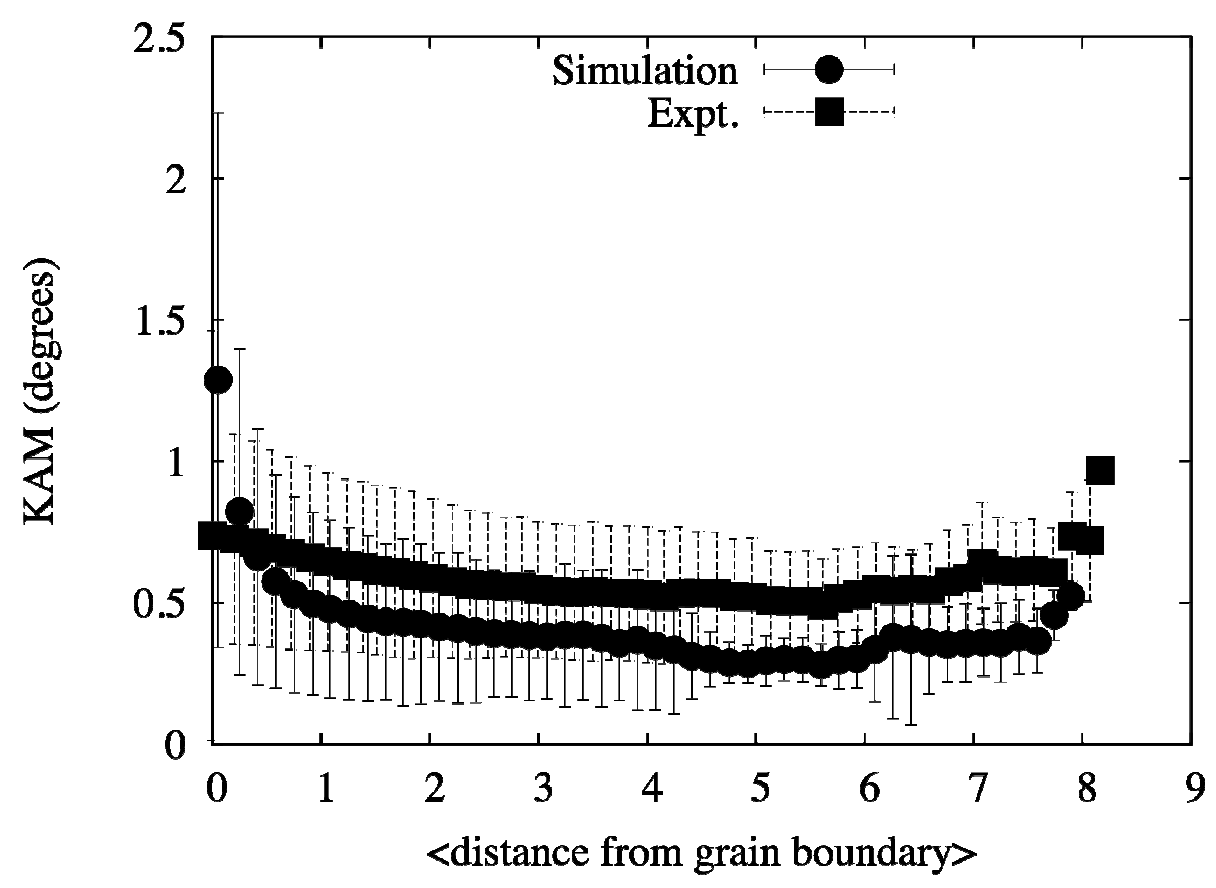

Fig. 4a. KAM, binned by grain boundary distance for both the experiment (Expt.) and the simulation (Simulation). As visual inspection of Fig. 3c suggests, large KAM values are found close to grain boundaries and vice versa. Data from simulation of tensile test to $12 \%$ strain. 


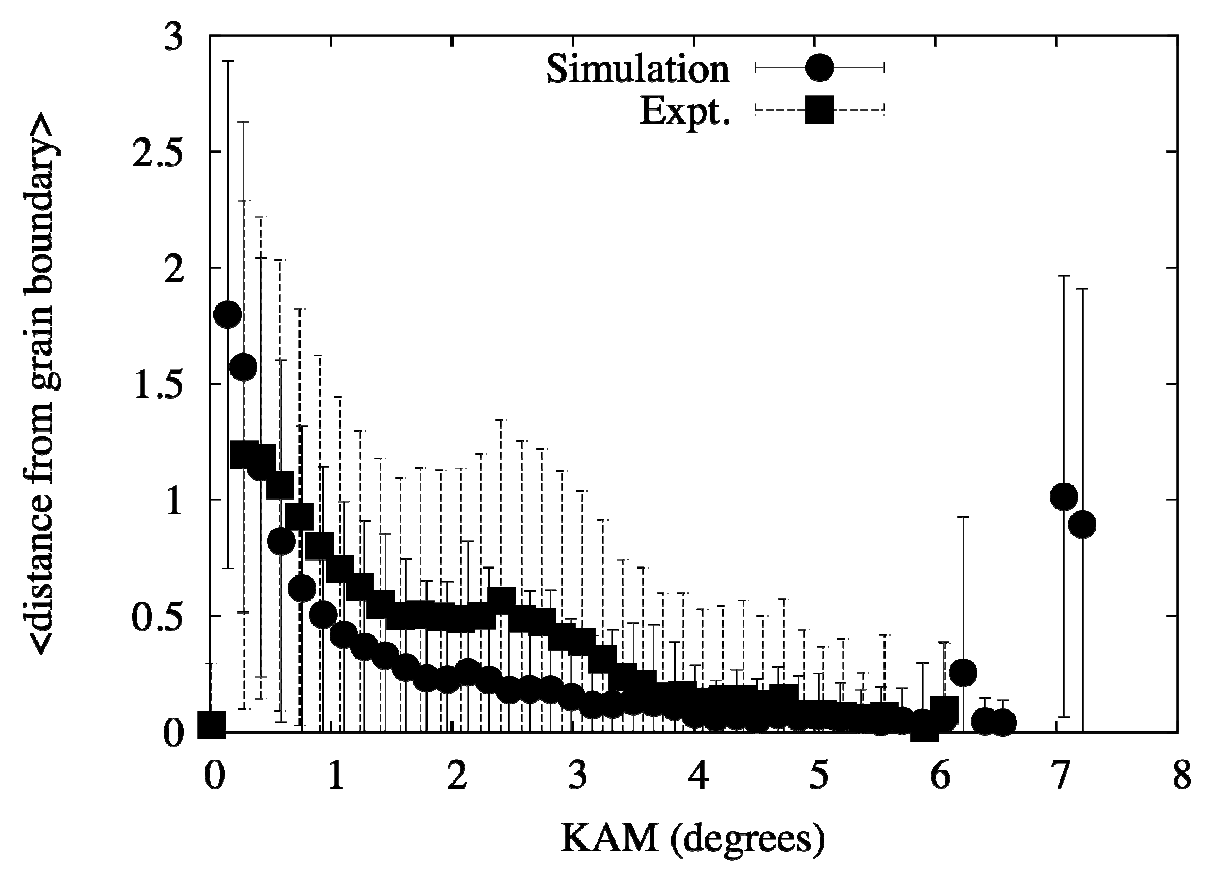

Fig. 4b. Grain

boundary

distance, binned

and averaged by

KAM value for

both the

experiment

(Expt.) and the

simulation

(Simulation).

The quantities

are all

normalized by

the global

average values.

The tensile

strain is $12 \%$.

Given the strong similarities between the local average misorientation and stress gradient maps, Figs. $3 \mathrm{c}$ and $3 \mathrm{~d}$, the same correlation was computed for this pair of variables. Fig. 5 shows a plot of KAM binned by stress gradient. The scatter in the KAM is approximately proportional to the values and a strong positive correlation is evident. For the highest values of stress gradient, the number of points in each bin tails off, leading to large variability. The reason for such a marked correlation may be found in the nature of restricted, dislocation-mediated slip. As discussed extensively in the literature, e.g. [11], multiple slip systems must be active in order for a material to deform plastically and conform to an externally imposed strain. Accordingly, the yield surface for a single crystal of a metal deforming by restricted glide has sharp corners where each corner represents a stress state that activates multiple slip systems. The classical Bishop-Hill analysis [12] shows that the corners or vertices that are relevant to strain-controlled boundary conditions activate six or eight slip systems. Variations in the orientation of grains control which particular vertex is active and what is the ratio of external stress (magnitude) to critical resolved shear stress, i.e. Taylor factor. With this in mind, the variation in Taylor factor and stress state from one grain to another inevitably introduces gradients in stress, particularly near grain boundaries. The resulting variations in stress may alter the exact combination of active slip systems that operates at any given point in the material. Any variation in the combination of slip activities will be reflected in the lattice rotation, thus leading to the development of orientation gradients.

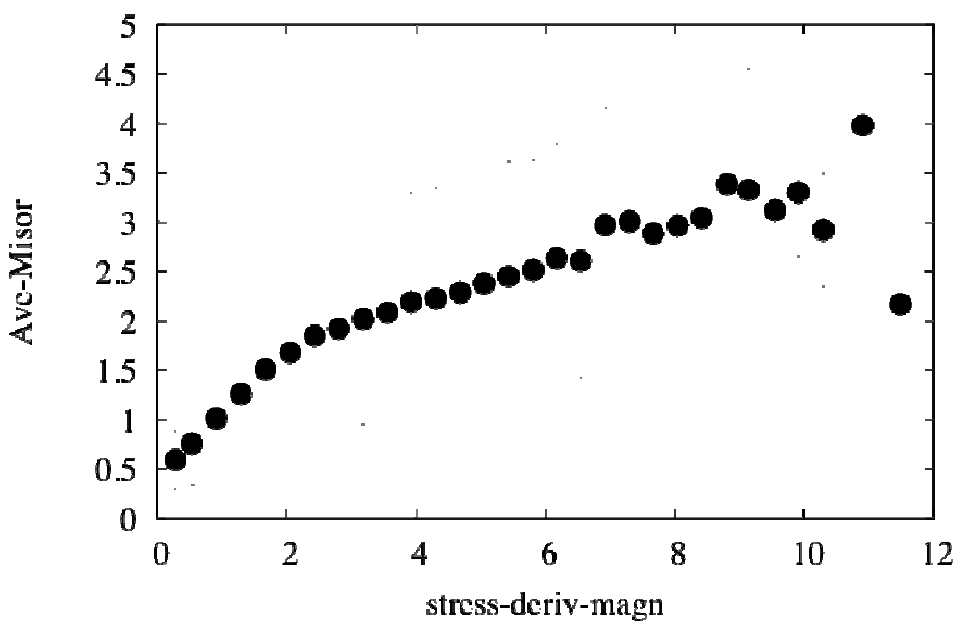

Fig. 5. Correlation plot of the KAM for points in a given range of the magnitude of the stress gradient. The error bars are drawn at \pm 1 standard deviation. Although the scatter at each point is substantial, the KAM is clearly positively correlated with the stress gradients in the system. Data obtained from simulation of $12 \%$ tensile strain. 


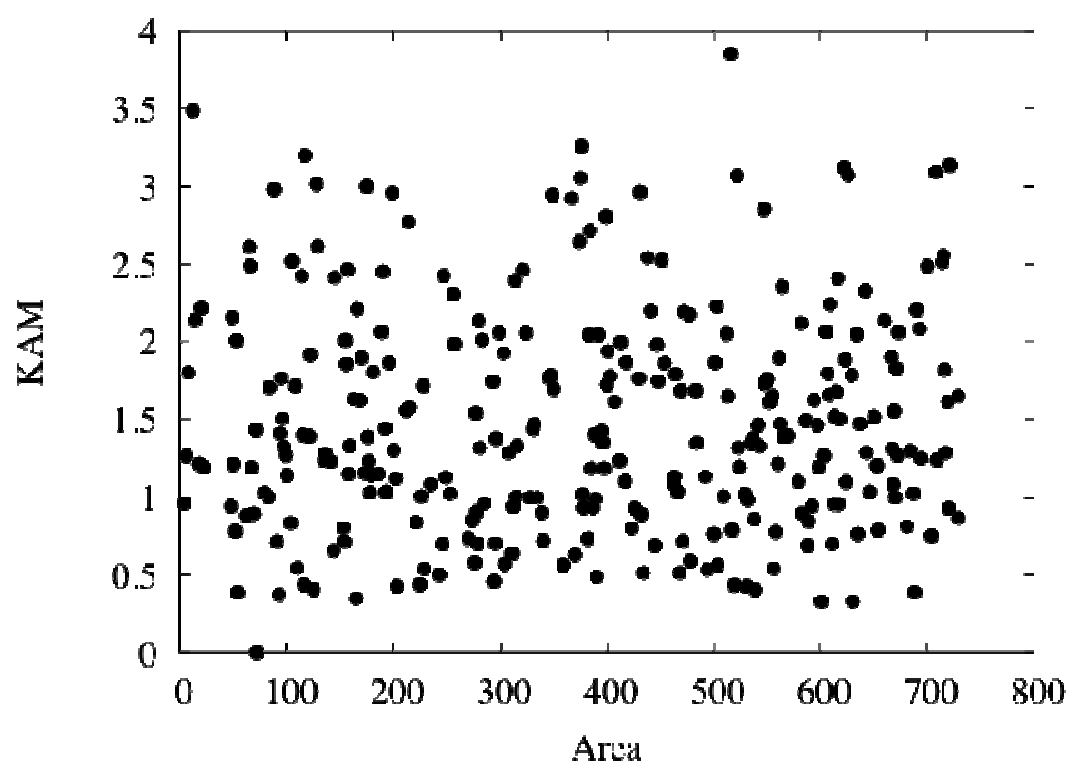

Fig. 6. Scatter plot of average value of KAM in each grain against the size of the grain (291 grains). No correlation between misorientation and grain size is evident.

Lastly, in order to check whether the development of orientation gradients is related to the grain size, Fig. 6 shows the KAM averaged over all points in each individual grain, plotted against the size of that grain. This shows no evidence for a trend with grain size, which is unsurprising since the constitutive model for rate-dependent crystal plasticity did not include any grain size dependent terms, as, for example, a Hall-Petch relationship would have.

\section{Conclusions and Summary}

The main results are (a) that orientation gradients concentrate near grain boundaries and (b) that there is a strong correlation between the existence of local orientation gradients and the presence of strong stress gradients. A reason for the correlations is offered in terms of crystal plasticity theory. Further research is indicated to quantify and rationalize the observed correlations. No relationship between orientation gradient and grain size was apparent, nor was any correlation with grain orientation found.

\section{References}

[1] C.S. Barrett, L.H. Levenson, Trans. Met. Soc. AIME, 137 (1940) 112-127.

[2] M. Ashby, Phil. Mag., 21 (1970) 399-424.

[3] B.S. El-Dasher, B.L. Adams, A.D. Rollett, Scripta mater., 48 (2003) 141-145.

[4] H. Moulinec, P. Suquet, Computer Methods in Applied Mechanics and Engineering, 157 (1998) 69-94.

[5] R.A. Lebensohn, Acta mater., 49 (2001) 2723-2737.

[6] R.A. Lebensohn, Y. Liu, P.P. Castaneda, Acta mater., 52 (2004) 5347-5361.

[7] R.A. Lebensohn, R. Brenner, O. Castelnau, A.D. Rollett, Acta mater., 56 (2008) 3914-3926.

[8] A.D. Rollett, R.A. Lebensohn, M. Groeber, Y. Choi, J. Li, G.S. Rohrer, MSMSE, 18 (2010).

[9] S.K. Mishra, P. Pant, K. Narasimhan, A.D. Rollett, I. Samajdar, Scripta mater., 61 (2009) 273276.

[10] T. Saito, J. Toriwaki, Ieice Transactions on Information and Systems, E77D (1994) 1005-1016. [11] U.F. Kocks, C. Tomé, H.-R. Wenk, in, Cambridge University Press, Cambridge, UK, 1998, pp. 675.

[12] J. Bishop, R. Hill, Phil. Mag., 42 (1951) 414-427. 